\title{
Consumption-Savings Decisions with Quasi-Geometric Discounting
}

\author{
Per Krusell and Anthony A. Smith, Jr. ${ }^{1}$
}

First version: June 1999

Current version: June 2001

\begin{abstract}
We study the consumption-savings problem of an infinitely-lived, rational consumer who has time-inconsistent preferences in the form of quasi-geometric discounting. The consumer operates a weakly concave production function and must simply divide current resources into current consumption and savings. There is no uncertainty. A solution to the consumer's problem is a subgame-perfect equilibrium of a dynamic game between the consumer's "successive selves." We show that this game has a continuum of equilibria in stationary Markov strategies. Not only is the stationary point of the consumer's asset holdings indeterminate, but each stationary point has a continuum of dynamic paths converging to it. The savings policy rules underlying these dynamic paths are step functions.
\end{abstract}

\footnotetext{
${ }^{1}$ Per Krusell's affiliations are the University of Rochester, Centre for Economic Policy Research, and the Institute for International Economic Studies. Anthony A. Smith, Jr.'s affiliation is Carnegie Mellon University. We would like to thank John H. Boyd III, Gino Gancia, Faruk Gul, Bård Harstad, Wolfgang Pesendorfer, three anonymous referees, and seminar participants at the NBER Summer Institute, Stanford University, and the University of Rochester for helpful comments. Both authors acknowledge financial support from the National Science Foundation.
} 


\section{Introduction}

The purpose of this paper is to study how an infinitely-lived consumer with "quasi-geometric" discounting - thought of as represented by a sequence of "selves" with conflicting preferences would make consumption and savings decisions. In light of experimental evidence suggesting that individuals do not have geometric discount functions (see, for example, Ainslie (1992) and Kirby and Herrnstein (1995)), it is important to understand how departures from geometric discounting affect an individual's consumption-saving decisions. ${ }^{1}$

We assume that time is discrete and that the consumer cannot commit to future actions. We assume that the consumer is rational in that he is able to forecast correctly his future actions: a solution to the decision problem is required to take the form of a subgame-perfect equilibrium of a game where the players are the consumer and his future selves. We restrict attention to equilibria which are stationary: they are Markov in current wealth; that is, current savings cannot depend either on time or on any other history than that summarized by current wealth.

The consumption-savings problem is of the simplest possible kind: there is no uncertainty, and current resources simply have to be divided into current consumption and savings. The period utility function is strictly concave, and the consumer operates a technology for saving which has (weakly) decreasing returns. A special case is that of an affine production function; this special case can be interpreted as one with a price-taking consumer who has a constant stream of labor income and can save at an exogenous interest rate.

Our main finding is one of indeterminacy of Markov equilibrium savings rules: there is a continuum of such rules. These rules differ both in their stationary points and in their implied dynamics. First, there is a continuum of implied stationary points to which the consumer's asset holdings may converge over time. Second, associated with each stationary point is a continuum of savings rules, implying that there is a continuum of dynamic paths converging to each stationary point. We construct these equilibria explicitly - the savings rules are step functions. The discontinuities in the step functions are key: payoff functions with jumps can be optimal precisely because the different selves have conflicting preferences, and depending on how the jumps are structured they can make one self behave more in the interest of another self or vice versa.

The indeterminacy in Markov strategies that we document in this paper is a new finding in the literature on consumption-savings decisions with quasi-geometric discounting. ${ }^{2}$ There is also a related literature on differential Markov games, e.g., in applications to models with imperfect altruism (see, e.g., Leininger (1986) and Bernheim and Ray (1987)) and resource extraction (see, e.g., Fudenberg and Tirole (1991)), where existence problems as well as multiplicity have been

\footnotetext{
${ }^{1}$ The idea that consumers do not discount the future geometrically, and, more generally, exhibit "time inconsistencies," originates with Strotz (1956). Pollak (1968), Phelps and Pollak (1968), Laibson (1994, 1997), and others have elaborated on this idea.

${ }^{2}$ Laibson (1994) and Bernheim, Ray, and Yeltekin (1999) find indeterminacy using history-dependent ("trigger") strategies. Harris and Laibson (2000a) provide an existence proof, but no results on multiplicity, for a consumptionsavings problem with uncertainty that does not nest ours. Harris and Laibson (2000b) provide a uniqueness result for a deterministic continuous-time consumption-savings model with "instantaneous gratification" (i.e., the consumer's departure from geometric discounting occurs only in the instantaneous present). Discussions of existence and uniqueness can also be found in Morris (2000) and in Morris and Postlewaite (1997).
} 
noted. That literature, however, did not uncover indeterminacy results. Moreover, our equilibrium construction here - whose key elements are discontinuities in decision rules and conflicting objective functions of the players at different points in time - actually suggests that indeterminacy of Markov equilibria can be present in a much larger class of problems than those we study here, including, e.g., optimal fiscal and monetary policy problems where the policy maker cannot commit to future policies.

We describe our model in Section 2. In Section 3 we provide the key elements of our equilibrium construction - the intuition underlying our result. We then provide the statement of our theorem in Section 4 and its proof in Section 5.

\section{The Model}

Time is discrete and infinite and begins at time 0 . There is no uncertainty. An infinitely-lived consumer derives utility from a stream of consumption at different dates. We assume that the consumer's self at time $t$ ranks consumption sequences according to

$$
u_{t}+\beta\left(\delta u_{t+1}+\delta^{2} u_{t+2}+\delta^{3} u_{t+3}+\cdots\right),
$$

where the variable $u_{t}$ denotes the number of utils at time $t$; these utils are derived from a function $u\left(c_{t}\right)$, where $c_{t}$ is consumption at time $t$. In other words, discounting is quasi-geometric: it is geometric across all dates except the current date. We assume that $\delta<1$ and $\beta<1{ }^{3}$ This formulation embodies an assumption of stationarity: discounting at any point in time has the form $1, \beta \delta, \beta \delta^{2}, \ldots$ It follows that there is a conflict between selves whenever $\beta \neq 1$; e.g., self $t$ compares consumption at $t+1$ and $t+2$ differently than does self $t+1$.

We assume that the period utility function $u(c)$ is strictly increasing, strictly concave, and continuously differentiable. The consumer's resource constraint is: $c+k^{\prime}=f(k)$, where $k$ is current and $k^{\prime}$ next period's capital holdings, $f$ is a strictly increasing, (weakly) concave, and continuously differentiable production function.

We use recursive methods to study Markov equilibria of the dynamic game between the consumer's different selves. This means that the consumer perceives future savings decisions to be given by a function $g(k): k_{t+1}=g\left(k_{t}\right)$. Note that $g$ is time-independent and that its only argument is current capital. The current self thus solves the "first-stage" problem

$$
\max _{k^{\prime}} u\left(f(k)-k^{\prime}\right)+\beta \delta V\left(k^{\prime}\right),
$$

where $V$ is an "indirect" utility function: it must satisfy the "second-stage" functional equation

$$
V(k)=u(f(k)-g(k))+\delta V(g(k)) .
$$

This equation incorporates the assumption that each of the consumer's future selves uses the same savings decision rule $g$. A Markov equilibrium obtains if $g(k)$ also solves the consumer's first-stage problem for all $k$.

\footnotetext{
${ }^{3}$ Our theorem could alternatively be stated also to include $\beta>1$, since indeterminacy applies in that case too; see footnote 4 below.
} 


\section{The Step-Function Equilibrium}

Here we describe the key elements of our equilibrium construction and provide some intuition. A detailed statement of the theorem and the proof of the theorem are provided in subsequent sections.

To construct a decision rule, select an arbitrary stationary point for the individual's capital holdings, $\bar{k}$ (we will see that, for some production functions, one needs to restrict the choice of $\bar{k}$ to a bounded range). We will now support $\bar{k}$ as a long-run outcome with a decision rule $g(k)$ which, for $k<\bar{k}$, is a step function with a countable number of steps indexed by $n$. Figure 1 illustrates a typical equilibrium step function $g .{ }^{4}$

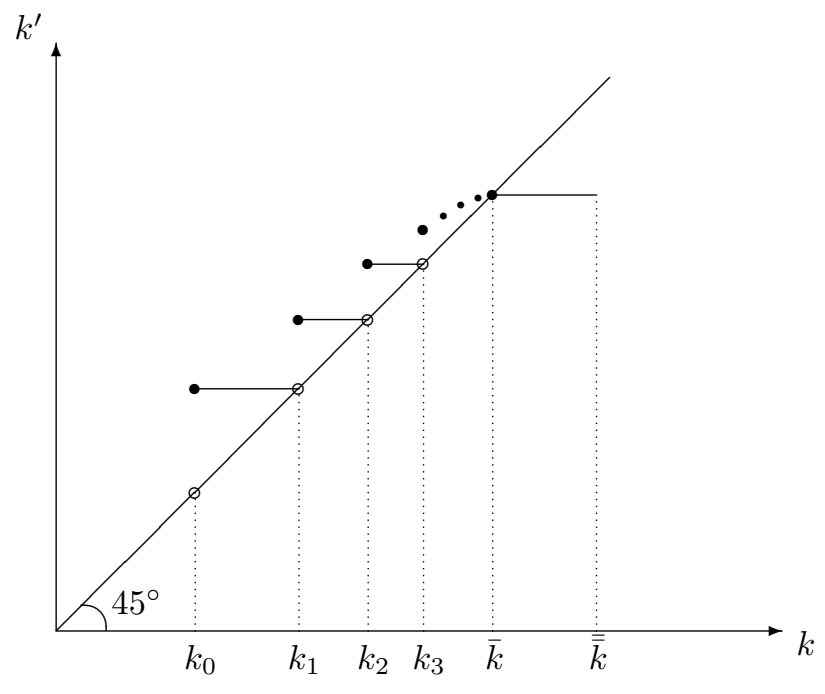

Figure 1

To construct the steps, we proceed as follows. First, select an arbitrary initial step $k_{0}<\bar{k}$ (in a neighborhood of $\bar{k}$ ) and form a step sequence by associating a level of capital with each step: $\left\{k_{n}\right\}_{n=0}^{\infty}$. The steps are defined by requiring that the current self be indifferent between remaining at $k_{n}$ and moving to the next level, $k_{n+1}$. The indifference is resolved in favor of higher saving: $g$ implies that, in every period, capital moves up one step. The sequence of step levels will thus also constitute the equilibrium outcome of the game. As we show below, the indifference requirement formally amounts to a second-order difference equation in the step sequence $\left\{k_{n}\right\}_{n=0}^{\infty}$. The construction of the step sequence thus involves solving this difference equation given an initial condition $k_{0}$ and a requirement of convergence to $\bar{k}$.

As we will now argue, this procedure involves a parametric restriction. When self 0 compares staying at a step and moving one step up, one can define, for any subsequent self $n$ along the resulting sequence, a corresponding "marginal propensity to save" by $\lambda_{n} \equiv\left(k_{n+2}-k_{n+1}\right) /\left(k_{n+1}-\right.$ $\left.k_{n}\right)$. This is the increase in self $n$ 's saving per unit of increase in his incoming capital that results from the current self moving up one step in saving. In the neighborhood of $\bar{k}$, the marginal propensity to save can be thought of as constant. Then the net present value return from taking

\footnotetext{
${ }^{4}$ When $\beta>1$, the steps are to the right of $\bar{k}$.
} 
the step is approximately $-1+\beta \delta\left(f^{\prime}-\lambda\right)+\beta \delta^{2}\left(f^{\prime}-\lambda\right) \lambda+\beta \delta^{3}\left(f^{\prime}-\lambda\right) \lambda^{2}+\ldots=-1+\beta \delta\left(f^{\prime}-\lambda\right) /(1-\delta \lambda)$ (the marginal utilities can be ignored since we are in a neighborhood of a stationary point). For the current self, then, to be indifferent between taking and not taking the step, we see that $\lambda$ has to equal $\left(1-\beta \delta f^{\prime}(\bar{k})\right) /(\delta(1-\beta))$. For our construction to work, this number cannot exceed one (then the steps do not converge) nor be below zero (then the sequence is not monotone). So we can only construct step-function equilibria for stationary points $\bar{k}$ such that $f^{\prime}(\bar{k}) \in\left(\frac{1}{\beta \delta}-\frac{1-\beta}{\beta}, \frac{1}{\beta \delta}\right)$. Clearly, $\beta=1$ only leaves one possible stationary point.

To show that $g$ prescribes optimal behavior given the perception that $g$ is used in the future, one needs to check all possible one-period deviations. The key elements in the argument, however, can be illustrated with one example: we will argue why it is locally optimal at $k_{n}$ to choose $k_{n+1}$, i.e., why self $n$, if given $k_{n}$, will leave self $n+1$ with $k_{n+1}$, as opposed to slightly higher or lower saving.

First, consider the possibility of saving slightly more, $k_{n+1}+\epsilon$, where $\epsilon$ is small and positive. According to the perceived step-function behavior of future selves, this deviation increases the consumption of self $n+1$ by the entire amount $\epsilon$ including its return, while at the same time leaving the future capital stocks unaltered. Thus, by this alternative choice, self $n$ would lower his consumption, increase the consumption of self $n+1$, and leave the consumption of all subsequent selves unaltered (perhaps consult Figure 1 again to see this). In terms of future capital stocks, we are thus comparing the equilibrium sequence $\left(k_{n}, k_{n+1}, k_{n+2}, k_{n+3}, \ldots\right)$ to the deviation $\left(k_{n}, k_{n+1}+\right.$ $\left.\epsilon, k_{n+2}, k_{n+3}, \ldots\right)$. Since whether this deviation is optimal is simply a matter of trading off current consumption against next period's consumption, lower values for $\epsilon$ are strictly better if $\beta \delta f^{\prime}(\bar{k})<1$. This condition is met by our above restrictions.

Turning to the possibility of slightly lower saving, we will now see how time-inconsistency is a necessary ingredient in supporting the step-function equilibrium. So suppose instead that $\epsilon$ is arbitrarily small but negative. This deviation causes next period's saving to fall discontinuously from $k_{n+1}$ to $k_{n}$ (again, imagine the deviation in Figure 1). Thus, all future consumption levels are affected, since we fall "one step behind" relative to when $\epsilon=0$ : we are now comparing the equilibrium sequence $\left(k_{n}, k_{n+1}, k_{n+2}, k_{n+3}, \ldots\right)$ to the deviation $\left(k_{n}, k_{n+1}-\epsilon, k_{n+1}, k_{n+2}, \ldots\right)$. Since current consumption is (almost) unaffected, we must decide whether a discontinuous increase in consumption in the next period and a resulting fall in consumption in the periods after that is a good deviation. It is not: on the equilibrium path, we have ensured that next period's self is indifferent between remaining on the current step and jumping up one step in saving. The current period's self, however, disagrees with this: he values saving strictly more, so long as $\beta<1$, since his discount rate between next period and the period after that is $\delta$, not $\beta \delta$, which is the rate used by his next self. Thus, stepping down in saving next period is a move in the wrong direction.

The previous arguments are also illustrated in Figure 2 where we plot the utility, as a function of the chosen savings $k^{\prime}$, for the current self who is endowed with $k_{n}$. 


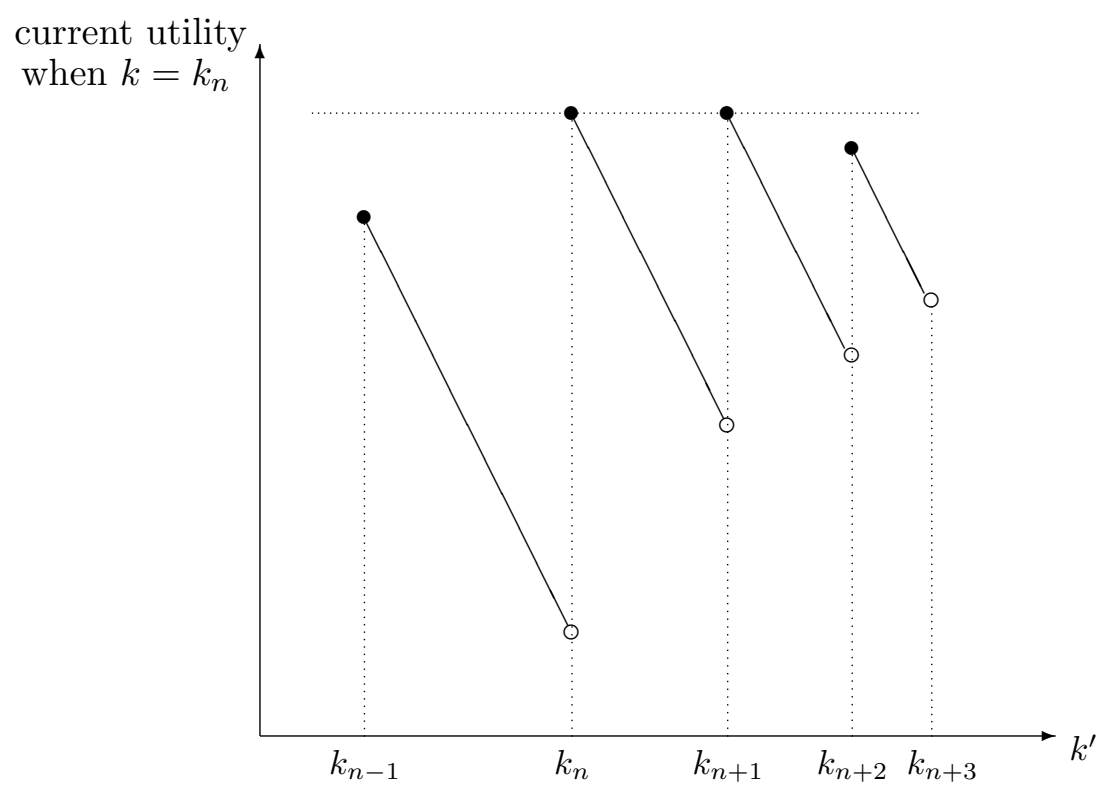

Figure 2

The features that are worth noting are: (i) the individual always chooses a "corner": choosing the left end points of any flat section always dominates choosing an interior point, because $\beta \delta f^{\prime}(\bar{k})<1$, and moving further left would cause a discontinuous utility fall; (ii) $k_{n}$ gives the same utility as $k_{n+1}$ (by construction); and (iii) all the other step points are dominated (this can be shown using strict concavity of $u$ ). Similar figures can be constructed if the individual starts at intermediate values for current capital. For an initial $k$ slightly above $k_{n}$, the values in the figure change so that saving $k_{n+1}$ now strictly dominates saving $k_{n}$ (and vice versa for initial values of $k$ slightly below $k_{n}$ : now $k_{n}$ is a strictly better choice).

Finally, one can easily extend the step function to the right of $\bar{k}$ with a flat section (thus implying convergence within one period) over a range. The idea here is the same: the individual goes to a corner.

The previous arguments are all straightforward to make in formal detail; we save this for the proof. Moreover, the arguments are of a rather general nature; their essential ingredient is how a disagreement between the two consecutive selves is used to support the discontinuous behavioral rule. This is why we suspect that this equilibrium construction can be used in a larger class of models.

\section{Statement of the Theorem}

For the construction of any equilibrium, we restrict the domain of capital to an interval $[\bar{k}-\epsilon, \overline{\bar{k}}]$, where $\bar{k}$ is a stationary point of the decision rule, $\epsilon$ is a small positive number (defining a leftneighborhood of $\bar{k}$ ), and $\overline{\bar{k}}$ is a constant greater than $\bar{k}$. We change the domain as we construct the continuum of equilibria with different stationary points.

Before stating a formal proposition summarizing our multiplicity result, we describe how to 
construct a typical decision rule and associated value function satisfying the equilibrium conditions discussed in Section 2.

The step function is defined by a countably infinite number of steps at the points $\left\{k_{n}\right\}_{n=0}^{\infty}$. This sequence is strictly increasing and converges to the stationary point $\bar{k}$. Given a sequence $\left\{k_{n}\right\}_{n=0}^{\infty}$, the step function $g$ for savings is thus defined by

$$
g(k)= \begin{cases}k_{n+1} & \text { if } k \in\left[k_{n}, k_{n+1}\right) \text { for some } n \in\{0,1,2, \ldots\} \\ \bar{k} & \text { if } k \in[\bar{k}, \overline{\bar{k}}]\end{cases}
$$

We need to find an appropriate sequence $\left\{k_{n}\right\}_{n=0}^{\infty}$. In order to specify the requirements that this sequence must satisfy, it is convenient to specify a sequence $\left\{v_{n}\right\}_{n=0}^{\infty}$ of values on the steps: $v_{n}=V\left(k_{n}\right)$; recall that $\beta \delta V\left(k^{\prime}\right)$ is the indirect utility, appropriate for the current self, of saving $k^{\prime}$. By definition, thus, the sequence $\left\{v_{n}\right\}_{n=0}^{\infty}$ satisfies

$$
v_{n}=u\left(f\left(k_{n}\right)-k_{n+1}\right)+\delta v_{n+1}, \quad n=0,1,2, \ldots
$$

The complete specification of $V$ on the domain $\left[k_{0}, \overline{\bar{k}}\right]$ therefore is as follows:

$$
V(k)= \begin{cases}u\left(f(k)-k_{n+1}\right)+\delta v_{n+1} & \text { if } k \in\left[k_{n}, k_{n+1}\right) \text { for some } n \in\{0,1,2, \ldots\} \\ u(f(k)-\bar{k})+\frac{\delta u(f(\bar{k})-\bar{k})}{1-\delta} & \text { if } k \in[\bar{k}, \overline{\bar{k}}]\end{cases}
$$

Now the step-function construction requires indifference on the steps: $\left\{k_{n}\right\}_{n=0}^{\infty}$ needs to satisfy

$$
u\left(f\left(k_{n}\right)-k_{n}\right)+\beta \delta v_{n}=u\left(f\left(k_{n}\right)-k_{n+1}\right)+\beta \delta v_{n+1}
$$

for $n=0,1,2, \ldots$. Thus, at $k_{n}$ the consumer saves $k_{n+1}$ for next period, but is indifferent between this choice and saving $k_{n}$.

The restrictions on $\left\{k_{n}\right\}_{n=0}^{\infty}$ can thus be stated as follows: it needs to be strictly increasing and to converge to $\bar{k}$ and there has to exist a sequence $\left\{v_{n}\right\}_{n=0}^{\infty}$ such that the two sequences satisfy the dynamic system given by equations (4) and (6).

Our result is thus as follows:

Theorem: Let $\bar{k}$ be any point satisfying

$$
1+\frac{1-\delta}{\beta \delta}<f^{\prime}(\bar{k})<\frac{1}{\beta \delta} .
$$

Then there is an $\epsilon>0$ defining a left-neighborhood of $\bar{k},(\bar{k}-\epsilon, \bar{k})$, such that for any $k_{0}$ in this neighborhood, there exist functions $g$ and $V$, defined by (3) and (5), and an associated stationary point $\bar{k}$, together defining an equilibrium to the multiple-selves game over the domain $\left[k_{0}, \overline{\bar{k}}\right]$, where $\overline{\bar{k}}>\bar{k}$ solves $u^{\prime}(f(\overline{\bar{k}})-\bar{k})=\beta \delta u^{\prime}(f(\bar{k})-\bar{k}) f^{\prime}(\bar{k})$.

Clearly, (7) defines a nontrivial interval so long as $\beta<1$ (as $\beta$ goes to 1 , the interval shrinks to one point).

The theorem states that $k_{0}$ can be chosen freely. This means that, since different choices for $k_{0}$ imply different step sequences, a given stationary point can be reached with a large set of different 
dynamic paths: we have an indeterminacy both in the set of stationary points and in the set of paths leading in to a given point.

In other work (Krusell, Kuruşçu, and Smith (2001)), we study solutions to the multiple-selves that feature a differentiable decision rule $g$-these are obtained as limits of equilibria in finitehorizon models. There, the key intertemporal condition on $g$ is the "generalized Euler equation":

$$
u^{\prime}\left(c_{t}\right)=\beta \delta u^{\prime}\left(c_{t+1}\right)\left\{f^{\prime}\left(k_{t+1}\right)+\left(\frac{1}{\beta}-1\right) g^{\prime}\left(k_{t+1}\right)\right\},
$$

where $g^{\prime}\left(k_{t+1}\right)$ denotes the marginal propensity to save next period, a quantity that cannot be ignored since the envelope theorem does not apply: the current self values saving next period $(1 / \beta$ times) more than the next-period self. One sees from this expression that if the local dynamics are monotone and stable, i.e., if $g^{\prime} \in(0,1)$, then $f^{\prime}$ has to lie somewhere in the range $\left(\frac{1}{\beta \delta}-\frac{1-\beta}{\beta}, \frac{1}{\beta \delta}\right)$. This is exactly the range for which we can construct step-function equilibria.

Our step-function equilibria also satisfy a generalized Euler equation of sorts, namely that which is derived from requiring indifference on any two adjacent steps:

$$
\begin{gathered}
\frac{u\left(f\left(k_{n}\right)-k_{n}\right)-u\left(f\left(k_{n}\right)-k_{n+1}\right)}{k_{n+1}-k_{n}}= \\
\beta \delta\left\{\frac{u\left(f\left(k_{n+1}\right)-k_{n+1}\right)-u\left(f\left(k_{n}\right)-k_{n+1}\right)}{f\left(k_{n+1}\right)-f\left(k_{n}\right)}\left(\frac{f\left(k_{n+1}\right)-f\left(k_{n}\right)}{k_{n+1}-k_{n}}\right)+\right. \\
\left.\left(\frac{1}{\beta}-1\right) \frac{u\left(f\left(k_{n+1}\right)-k_{n+1}\right)-u\left(f\left(k_{n+1}\right)-k_{n+2}\right)}{k_{n+2}-k_{n+1}}\left(\frac{k_{n+2}-k_{n+1}}{k_{n+1}-k_{n}}\right)\right\} .
\end{gathered}
$$

This equation, which follows from equations (4) and (6), is our second-order difference equation in $k_{n}$. Its structure is similar to that of the generalized Euler equation for differentiable $g$ 's. Defining $c_{n} \equiv f\left(k_{n}\right)-k_{n+1}$, it says approximately that:

$$
u^{\prime}\left(c_{n}\right)=\beta \delta\left\{u^{\prime}\left(c_{n}\right) f^{\prime}\left(k_{n}\right)+\left(\frac{1}{\beta}-1\right) u^{\prime}\left(c_{n+1}\right) \lambda_{n}\right\} .
$$

In other words, the current disutility of saving more has to equal the discounted gain from increased production next period (the first term on the right-hand side) and increased saving next period (the second term on the right-hand side). ${ }^{5}$

Along a differentiable path, Krusell, Kuruşçu, and Smith (2001) show that decentralized (pricetaking) and centralized (home-production) environments give different outcomes: the former give higher savings rates, and higher steady states. Under isoelastic utility, one can show that the competitive equilibrium steady state is that which would result with a time-consistent agent with a discount rate of precisely $\frac{\beta \delta}{1-\delta(1-\beta)}$ : our highest steady state above. Thus, with step functions, it is possible to attain at most the price-taking steady state. The highest steady state obtainable with step functions and a decreasing-returns $f$ is therefore higher than the corresponding differentiable- $g$ steady state. Moreover, one can show that the latter is dominated in utility - as evaluated by the current self-by a step-function equilibrium with higher savings.

\footnotetext{
${ }^{5}$ As $k_{n}$ approaches a steady state, one sees as well that $1=\beta \delta\left(f^{\prime}+\left(\frac{1}{\beta}-1\right) \lambda\right)$. Solving for $f^{\prime}$ for $\lambda=0$ and $\lambda=1$ delivers the restrictions on $f^{\prime}$ stated in the theorem.
} 
If $f$ is affine, we are in fact considering a consumer who can save at a gross rate $R$ and has exogenous (perhaps labor) income $w$. In that case, our theorem applies so long as $R$ is in a certain range (replace $f^{\prime}(\bar{k})$ by $R$ in $(7)$ ). If it is, then any value for capital is a stationary point. Thus, the indeterminacy in steady states that obtains in the standard model when the interest rate exactly equals the discount rate obtains here for a range of interest rates.

In a representative-agent, general-equilibrium economy, condition (7) specifies a range of steadystate capital stocks. Thus, suppose that capital is in this range, thus delivering an interest rate $R$ in this range. Then it is optimal for the agent to choose any capital stock, in particular the selected one, so we indeed have a steady-state general equilibrium.

\section{Proof of the Theorem}

To construct the sequences $\left\{k_{n}\right\}_{n=0}^{\infty}$ and $\left\{v_{n}\right\}_{n=0}^{\infty}$, let $\bar{v}$ be the stationary point implied by $\bar{k}$ being the stationary point of the dynamic system given by equations (4) and $(6): \bar{v}=\frac{u(f(\bar{k})-\bar{k})}{1-\delta}$. The dynamic system (4) and (6) has to involve convergence to $(\bar{k}, \bar{v})$, and the convergence for capital has to be monotone increasing. Equations (4) and (6) define an implicit function that maps $\left(k_{n}, v_{n}\right)$ into $\left(k_{n+1}, v_{n+1}\right)$ whose Jacobian matrix of first derivatives, evaluated at a stationary point $(\bar{k}, \bar{v})$, has one eigenvalue equal to 1 and one eigenvalue equal to $\frac{1-\beta \delta f^{\prime}(\bar{k})}{\delta(1-\beta)}$. This eigenvalue is between 0 and 1 provided that $1+\frac{1-\delta}{\beta \delta}<f^{\prime}(\bar{k})<\frac{1}{\beta \delta}$. This condition is satisfied by the assumption in the theorem. Under this condition, it is straightforward to modify standard results concerning the local stability of nonlinear difference equations (see, e.g., Scheinkman (1973)) to show that the dynamic system given by (4) and (6) has a one-dimensional stable manifold characterized by a continuously differentiable function $\varphi\left(k_{0}, v_{0}\right)$. In other words, given a stationary point $(\bar{k}, \bar{v})$, there exists a neighborhood $N$ of this point such that the dynamic system (4) and (6) converges, monotonically, to the stationary point for any initial value $\left(k_{0}, v_{0}\right) \in N$ satisfying $\varphi\left(k_{0}, v_{0}\right)=0$. We can, therefore, construct a continuum of sequences $\left\{\left(k_{n}, v_{n}\right)\right\}$ that converge to a given stationary point. Each of these sequences determines a decision rule and a value function that satisfy the equilibrium conditions in Section 2.

As described in Section 4, given the decision rule $g$ defined by equation (3), the value function $V$ defined by equation (5) satisfies the second-stage functional equation (2) by construction. To check that $g$ solves the first-stage problem (1) given $V$, four lemmas will be stated and proved. Each lemma considers a specific kind of deviation from the proposed decision rule.

Lemma 1: For any $k \in\left[k_{0}, \overline{\bar{k}}\right]$, any choice $k^{\prime} \in\left(k_{n}, k_{n+1}\right)$, for some $n$, or $k^{\prime} \in[\bar{k}, \overline{\bar{k}})$, can be improved upon.

Proof: Given that $\beta \delta f^{\prime}(\bar{k})<1$, it is always strictly better to be at the left endpoint of an interval than in the interior. For $k \in[\bar{k}, \overline{\bar{k}}]$, the proof of this statement is immediate. For $k \in\left[k_{0}, \bar{k}\right)$, we use the fact that $\beta \delta f^{\prime}(k)<1$ when $k$ is sufficiently close to $\bar{k}$.

Lemma 2: For $k \in[\bar{k}, \overline{\bar{k}}], k^{\prime}=\bar{k}$ dominates $k^{\prime}=k_{n}$ for all $n$.

Proof: We need to prove that $u(f(k)-\bar{k})-u\left(f(k)-k_{n}\right) \geq \beta \delta\left(v_{n}-\bar{v}\right)$. The left-hand side of this 
expression can be written

$$
\sum_{s=0}^{N}\left[u\left(f(k)-k_{n+s+1}\right)-u\left(f(k)-k_{n+s}\right)\right]+u(f(k)-\bar{k})-u\left(f(k)-k_{n+N+1}\right)
$$

which, since the last two terms cancel as $N$ goes to $\infty$, equals

$$
\sum_{s=0}^{\infty}\left[u\left(f(k)-k_{n+s+1}\right)-u\left(f(k)-k_{n+s}\right)\right]
$$

The right-hand side of the expression, in turn, can be rewritten as $\beta \delta \sum_{s=0}^{\infty}\left[v_{n+s}-v_{n+s+1}\right]$, since $v_{n}$ goes to $\bar{v}$ as $n$ goes to $\infty$. Using indifference on the steps, this expression becomes

$$
\sum_{s=0}^{\infty}\left[u\left(f\left(k_{n+s}\right)-k_{n+s+1}\right)-u\left(f\left(k_{n+s}\right)-k_{n+s}\right)\right] .
$$

It is now clear that the left-hand side is no less than the right-hand side if

$$
u\left(f\left(k_{n+s}\right)-k_{n+s}\right)-u\left(f\left(k_{n+s}\right)-k_{n+s+1}\right) \geq u\left(f(k)-k_{n+s}\right)-u\left(f(k)-k_{n+s+1}\right),
$$

for each $s \geq 0$. But from the strict concavity of $u$ these inequalities are all met (strictly), since $k>k_{n+s}$ and $\left\{k_{n}\right\}$ is a strictly increasing sequence.

Lemma 3: For all $n$, for $k \in\left[k_{n}, k_{n+1}\right), k^{\prime}=k_{n+s}$ for any $s>1$ or $s<0$ is dominated by $k^{\prime}=k_{n+1}$. Proof: We start with $s>1$. We need to show that $u\left(f(k)-k_{n+1}\right)-u\left(f(k)-k_{n+s}\right) \geq \beta \delta\left(v_{n+s}-\right.$ $\left.v_{n+1}\right)$. The left-hand side of this expression can be written

$$
\sum_{v=1}^{s-1}\left[u\left(f(k)-k_{n+v}\right)-u\left(f(k)-k_{n+v+1}\right)\right]
$$

and the right-hand side can be written $\beta \delta \sum_{v=1}^{s-1}\left[v_{n+v+1}-v_{n+v}\right]$, which from indifference at steps equals

$$
\sum_{v=1}^{s-1}\left[u\left(f\left(k_{n+v}\right)-k_{n+v}\right)-u\left(f\left(k_{n+v}\right)-k_{n+v+1}\right)\right] .
$$

Due to strict concavity of $u$ and the sequence $\left\{k_{n}\right\}$ being strictly increasing,

$$
\left.u\left(f(k)-k_{n+v}\right)-u\left(f(k)-k_{n+v+1}\right)>u\left(f\left(k_{n+v}\right)-k_{n+v}\right)-u\left(f\left(k_{n+v}\right)-k_{n+v+1}\right)\right]
$$

for each $v>0$. This implies that the left-hand side exceeds the right-hand side. When $s<0$, by indifference at the initial step, it suffices to show that $u\left(f(k)-k_{n}\right)-u\left(f(k)-k_{n-s}\right) \geq \beta \delta\left(v_{n-s}-v_{n}\right)$. Now form the same type of sums as for the $s>1$ case, but from $\nu=0$ to $s-1$, and proceed with an analogous argument.

Lemma 4: For all $n$, for $k \in\left[k_{n}, k_{n+1}\right), k^{\prime}=\bar{k}$ is dominated by $k^{\prime}=k_{n+1}$.

Proof: Noting that

$$
u\left(f\left(k_{n}\right)-k_{n+1}\right)-u\left(f\left(k_{n}\right)-\bar{k}\right)=\sum_{s=1}^{\infty}\left[u\left(f\left(k_{n}\right)-k_{n+s}\right)-u\left(f\left(k_{n}\right)-k_{n+s+1}\right)\right]
$$


and that

$$
\beta \delta\left(\bar{v}-v_{n+1}\right)=\sum_{s=1}^{\infty}\left(v_{n+s+1}-v_{n+s}\right)=\sum_{s=1}^{\infty}\left[u\left(f\left(k_{n+s}\right)-k_{n+s}\right)-u\left(f\left(k_{n}\right)-k_{n+s+1}\right)\right],
$$

the result again follows, using concavity and $\left\{k_{n}\right\}$ being an increasing sequence: this results in term-by-term domination.

Lemmas 1-4 suffice to support optimality of the constructed function $g$ on all of its domain.

\section{REFERENCES}

Ainslie, G.W. (1992), Picoeconomics, Cambridge: Cambridge University Press.

Bernheim, D.B. and D. Ray (1987), "Economic Growth with Intergenerational Altruism," Review of Economic Studies 53, 349-367.

Bernheim, D.B., D. Ray, and S. Yeltekin (1999), "Self-Control, Saving, and the Low Asset Trap," manuscript.

Fudenberg, D. and J. Tirole (1991), Game Theory, Cambridge: MIT Press.

Kirby, K.N. and R.J. Herrnstein (1995), "Preference Reversals Due to Myopic Discounting of Delayed Reward," Psychological Science 6, 83-89.

Krusell, P., B. Kuruş̧u, and A.A. Smith, Jr. (2001), "Equilibrium Welfare and Government Policy with Quasi-Geometric Discounting," manuscript.

Laibson, D. (1994), "Self-Control and Saving," Ph.D. dissertation (Massachusetts Institute of Technology).

Laibson, D. (1997), "Hyperbolic Discount Functions and Time Preference Heterogeneity," manuscript.

Laibson, D. and C. Harris (2000a), "Dynamic Choices of Hyperbolic Consumers," forthcoming in Econometrica.

Laibson, D. and C. Harris (2000b), "Instantaneous Gratification," manuscript.

Leininger, W. (1986), "The Existence of Perfect Equilibria in a Model of Growth with Altruism between Generations," Review of Economic Studies 53, 349-367.

Morris, S. (2000), "Notes on Three Period Intuition for the Cake Eating Problem with NonExponential Discounting," manuscript.

Morris, S. and A. Postlewaite (1997), "Observational Implications of Non-Exponential Discounting," manuscript.

Phelps, E.S. and R.A. Pollak (1968), "On Second-best National Saving and Game-equilibrium Growth," Review of Economic Studies, 35, 185-199.

Pollak, R.A. (1968), "Consistent Planning," Review of Economic Studies 35, 201-208.

Scheinkman, J.A. (1973), "On Optimal Steady States of $n$-Sector Growth Models When Utility is Discounted," Ph.D. dissertation (University of Rochester).

Strotz, R.H. (1956), "Myopia and Inconsistency in Dynamic Utility Maximization," Review of Economic Studies 23, 165-180. 